\title{
Deadbeat observer: construction via sets
}

\author{
S. Emre Tuna*
}

October 30, 2018

\begin{abstract}
A geometric generalization of discrete-time linear deadbeat observer is presented. The proposed method to generate a deadbeat observer for a given nonlinear system is constructive and makes use of sets that can be computed iteratively. For demonstration, derivations of observer dynamics are provided for various example systems. Based on the method, a simple algorithm that computes the deadbeat gain for a linear system with scalar output is given.
\end{abstract}

\section{Introduction}

Observer design for linear systems is generally acknowledged to be understood well enough. For discrete-time linear system $x^{+}=A x$ with output $y=C x$, Luenberger observer [8] dynamics read

$$
\hat{x}^{+}=A \hat{x}+L(y-C \hat{x})
$$

and designing the observer is nothing but choosing an observer gain $L$ that places the eigenvalues of matrix $A-L C$ within the unit circle. Simple and elegant, anyone would hardly doubt that this construction is the construction for linear systems. However, perhaps due arguably to over-elegance of the notation, it is nontrivial to unearth the true mechanism (if it exists) running behind Luenberger observer in order to generalize it in some natural way for nonlinear systems. In this paper we aim to provide a geometric interpretation of the righthand side of (10) for the particular case where matrix $A-L C$ is nilpotent, i.e., when the observer is deadbeat. Our interpretation allows one to construct deadbeat observers for nonlinear systems provided that certain conditions (Assumption 1 and Assumption 21) hold. We now note and later demonstrate that when the system is linear those assumptions are minimal for a deadbeat observer to exist. The literature on observers accommodates significant results. See, for instance, 5, 4, 9, 13, 11, 3, 1, 14.

${ }^{*}$ Author is with Department of Electrical and Electronics Engineering, Middle East Technical University, 06800 Ankara, Turkey. Email: tuna@eee.metu.edu.tr 
The toy example that we keep in the back of our mind while we attempt to reach a generalization is the simple case where $A$ is a rotation matrix in $\mathbb{R}^{2}$

$$
A=\left[\begin{array}{rr}
\cos \theta & -\sin \theta \\
\sin \theta & \cos \theta
\end{array}\right]
$$

with angle of rotation $\theta$ different from 0 and $\pi$. Letting $y=x_{2}$, i.e., $C=\left[\begin{array}{ll}0 & 1\end{array}\right]$, the deadbeat observer turns out to be

$$
\hat{x}^{+}=A \hat{x}+\left[\begin{array}{l}
\cos 2 \theta / \sin \theta \\
\sin 2 \theta / \sin \theta
\end{array}\right](y-C \hat{x})
$$

which can be rewritten as

$$
\hat{x}^{+}=A\left(\hat{x}+\left[\begin{array}{c}
\cot \theta \\
1
\end{array}\right](y-C \hat{x})\right)
$$

Now we state the key observation in this paper: The term in brackets is the intersection of two equivalence classes (sometimes called congruence classes [6]). Namely,

$$
\hat{x}+\left[\begin{array}{c}
\cot \theta \\
1
\end{array}\right](y-C \hat{x})=(\hat{x}+A \operatorname{null}(C)) \cap(x+\operatorname{null}(C))
$$

as shown in Fig. 1, Based on this observation, one contribution of this paper



Figure 1: Intersection of two equivalence classes.

is intended to be in showing that such equivalence classes can be defined even for nonlinear systems of arbitrary order, which in turn allows one to construct deadbeat observers. There is another possible contribution that is of more practical nature: We present a simple algorithm that computes, for linear systems with scalar output, deadbeat gain $L$ by iteratively intersecting linear subspaces. (Devising reliable numerical techniques to compute deadbeat gain for discretetime linear systems had once been an active field of research; see, for instance, [2, 7, 12.) 
The remainder of the paper is organized as follows. Next section contains some preliminary material. In Section 3 we give the formal problem definition. Section 4 is where we describe the sets that we use in construction of the deadbeat observer. We state and prove the main result in Section 5 An extension of the main result where we consider the case with input $\left(x^{+}=f(x, u)\right)$ is in Section 6. We provide examples in Section 7. where we construct deadbeat observers for two different third order systems. In Section 8 we present an algorithm to compute the deadbeat observer gain for a linear system with scalar output.

\section{Preliminaries}

Identity matrix is denoted by $I$. Null space and range space of a matrix $M \in$ $\mathbb{R}^{m \times n}$ are denoted by $\mathcal{N}(M)$ and $\mathcal{R}(M)$, respectively. Given map $\mu: \mathcal{X} \rightarrow \mathcal{Y}$, $\mu^{-1}(\cdot)$ denotes the inverse map in the general sense that for $y \in \mathcal{Y}, \mu^{-1}(y)$ is the set of all $x \in \mathcal{X}$ satisfying $\mu(x)=y$. That is, we will not need $\mu$ be bijective when talking about its inverse. Note that $y \notin \mu(\mathcal{X})$ will imply $\mu^{-1}(y)=\emptyset$. Linear maps $x \mapsto M x$ will not be exempt from this notation. The reader should not think that $M$ is a nonsingular matrix when we write $M^{-1} y$. (In our case $M$ need even not be square.) For instance, for $M=\left[\begin{array}{ll}0 & 0\end{array}\right]$ we have $M^{-1} y=\emptyset$ for $y \neq 0$ and $M^{-1} 0=\mathbb{R}^{2}$. The set of nonnegative integers is denoted by $\mathbb{N}$ and $\mathbb{R}_{>0}$ denotes the set of strictly positive real numbers.

\section{Problem definition}

Consider the following discrete-time system

$$
\begin{aligned}
x^{+} & =f(x) \\
y & =h(x)
\end{aligned}
$$

where $x \in \mathcal{X} \subset \mathbb{R}^{n}$ is the state, $x^{+}$is the state at the next time instant, and $y \in \mathcal{Y} \subset \mathbb{R}^{m}$ is the output or the measurement. The solution of system (2) at time $k \in \mathbb{N}$, starting at initial condition $x \in \mathcal{X}$ is denoted by $\phi(k, x)$. Note that $\phi(0, x)=x$ and $\phi(k+1, x)=f(\phi(k, x))$ for all $x$ and $k$.

Now consider the following cascade system

$$
\begin{aligned}
& x^{+}=f(x) \\
& \hat{x}^{+} \in g(\hat{x}, h(x))
\end{aligned}
$$

We denote $a$ solution of subsystem (3b) by $\psi(k, \hat{x}, x)$. We then have $\psi(0, \hat{x}, x)=$ $\hat{x}$ and $\psi(k+1, \hat{x}, x) \in g(\psi(k, \hat{x}, x), h(\phi(k, x)))$ for all $x, \hat{x}$, and $k$. We now use (3) to define deadbeat observer.

Definition 1 Given $g: \mathcal{X} \times \mathcal{Y} \rightrightarrows \mathcal{X}$, system

$$
\hat{x}^{+} \in g(\hat{x}, y)
$$


is said to be a deadbeat observer for system (2) if there exists $p \geq 1$ such that all solutions of system (3) satisfy

$$
\psi(k, \hat{x}, x)=\phi(k, x)
$$

for all $x, \hat{x} \in \mathcal{X}$ and $k \geq p$.

Definition 2 System (21) is said to be deadbeat observable if there exists a deadbeat observer for it.

In this paper we present a procedure to construct a deadbeat observer for system (2) provided that certain conditions (Assumption 1 and Assumption 2) hold. Our construction will make use of some sets, which we define in the next section. Before moving on into the next section, however, we choose to remind the reader of a standard fact regarding the observability of linear systems. Then we provide a Lemma 1 as a geometric equivalent of that well-known result. Lemma 1 will find use later when we attempt to interpret and display the generality of the assumptions we will have made.

The following criterion, known as Popov-Belevitch-Hautus (PBH) test, is an elegant tool for checking (deadbeat) observability.

Proposition 1 (PBH test) The linear system

$$
\begin{aligned}
x^{+} & =A x \\
y & =C x
\end{aligned}
$$

with $A \in \mathbb{R}^{n \times n}$ and $C \in \mathbb{R}^{m \times n}$ is deadbeat observable if and only if

$$
\operatorname{rank}\left[\begin{array}{c}
A-\lambda I \\
C
\end{array}\right]=n \quad \text { for all } \quad \lambda \neq 0
$$

where $\lambda$ is a complex scalar.

The below result is a geometric equivalent of PBH test.

Lemma 1 Given $A \in \mathbb{R}^{n \times n}$ and $C \in \mathbb{R}^{m \times n}$, let subspace $\mathcal{S}_{k}$ of $\mathbb{R}^{n}$ be defined as $\mathcal{S}_{k}:=A \mathcal{S}_{k-1} \cap \mathcal{S}_{0}$ for $k=1,2, \ldots$ with $\mathcal{S}_{0}:=\mathcal{N}(C)$. Then system (4) is deadbeat observable if and only if

$$
\mathcal{S}_{n}=\{0\} .
$$

Proof. For simplicity we provide the demonstration for the case where each $\mathcal{S}_{k}$ is a subspace of $\mathbb{C}^{n}$ (over field $\mathbb{C}$ ). The case $\mathcal{S}_{k} \subset \mathbb{R}^{n}$ is a little longer to prove yet it is true.

We first show (6) $\Longrightarrow$ (5). Suppose (5D) fails. That is, there exists an eigenvector $v \in \mathbb{C}^{n}$ and a nonzero eigenvalue $\lambda \in \mathbb{C}$ such that $A v=\lambda v$ and $C v=0$. Now suppose for some $k$ we have $v \in \mathcal{S}_{k}$. Then, since $v$ is an eigenvector with a nonzero eigenvalue, we can write $v \in A \mathcal{S}_{k}$. Observe that $v \in \mathcal{S}_{0}$ for $C v=0$. 
As a result $v \in A \mathcal{S}_{k} \cap \mathcal{S}_{0}=\mathcal{S}_{k+1}$. By induction therefore we have $v \in \mathcal{S}_{k}$ for all $k$, which means that (6) fails.

Now we demonstrate the other direction (5) $\Longrightarrow$ (6). We first claim that $\mathcal{S}_{k+1} \subset \mathcal{S}_{k}$ for all $k$. We use induction to justify our claim. Suppose $\mathcal{S}_{k+1} \subset \mathcal{S}_{k}$ for some $k$. Then we can write

$$
\begin{aligned}
\mathcal{S}_{k+2} & =A \mathcal{S}_{k+1} \cap \mathcal{S}_{0} \\
& \subset A \mathcal{S}_{k} \cap \mathcal{S}_{0} \\
& =\mathcal{S}_{k+1} .
\end{aligned}
$$

Since $\mathcal{S}_{1} \subset \mathcal{S}_{0}$ our claim is valid. A trivial implication of our claim then follows: $\operatorname{dim} \mathcal{S}_{k+1} \leq \operatorname{dim} \mathcal{S}_{k}$ for all $k$. Let us now suppose (6) fails. That is, $\operatorname{dim} \mathcal{S}_{n} \geq 1$. Note that $\operatorname{dim} \mathcal{S}_{0} \leq n$. Therefore $\operatorname{dim} \mathcal{S}_{n} \geq 1$ and $\operatorname{dim} \mathcal{S}_{k+1} \leq \operatorname{dim} \mathcal{S}_{k}$ imply the existence of some $\ell \in\{0,1, \ldots, n-1\}$ such that $\operatorname{dim} \mathcal{S}_{\ell+1}=\operatorname{dim} \mathcal{S}_{\ell} \geq 1$. Since $\mathcal{S}_{\ell+1} \subset \mathcal{S}_{\ell}$, both $\mathcal{S}_{\ell+1}$ and $\mathcal{S}_{\ell}$ having the same dimension implies $\mathcal{S}_{\ell+1}=\mathcal{S}_{\ell}$. Hence we obtained $\mathcal{S}_{\ell}=A \mathcal{S}_{\ell} \cap \mathcal{S}_{0}$ which allows us to write $\mathcal{S}_{\ell} \subset A \mathcal{S}_{\ell}$. Since $\operatorname{dim} \mathcal{S}_{\ell} \geq \operatorname{dim} A \mathcal{S}_{\ell}$ we deduce that $\mathcal{S}_{\ell}=A \mathcal{S}_{\ell}$. Since $\operatorname{dim} \mathcal{S}_{\ell} \geq 1$, equality $A \mathcal{S}_{\ell}=$ $\mathcal{S}_{\ell}$ implies that there exists an eigenvector $v \in \mathcal{S}_{\ell}$ and a nonzero eigenvalue $\lambda \in \mathbb{C}$ such that $A v=\lambda v$. Note also that $C v=0$ because $\mathcal{S}_{\ell} \subset \mathcal{S}_{0}$. Hence (5) fails.

Remark 1 It is clear from the proof that if (6) fails then $\operatorname{dim} \mathcal{S}_{k} \geq 1$ for all $k$.

\section{Sets}

In this section we define certain sets (more formally, equivalence classes) associated with system (2). For $x \in \mathcal{X}$ we define

$$
[x]_{0}:=h^{-1}(h(x)) .
$$

Note that when $h(x)=C x$, where $C \in \mathbb{R}^{m \times n}$, we have $[x]_{0}=x+\mathcal{N}(C)$. We then let for $k=0,1, \ldots$

$$
[x]_{k+1}:=[x]_{k}^{+} \cap[x]_{0}
$$

where

$$
[x]_{k}^{+}:=f\left(\left[f^{-1}(x)\right]_{k}\right) .
$$

Note that $[x]_{k}^{+}=\emptyset$ when $x \notin f(\mathcal{X})$ since then $f^{-1}(x)=\emptyset$.

Remark 2 Note that $[x]_{k+1} \subset[x]_{k}$ and $[x]_{k+1}^{+} \subset[x]_{k}^{+}$for all $x$ and $k$.

The following two assumptions will be invoked in our main theorem. In hope of making them appear somewhat meaningful and revealing their generality we provide the conditions that they would boil down to for linear systems. 
Assumption 1 There exists $p \geq 1$ such that, for each $x \in \mathcal{X}$, set $[x]_{p-1}$ is either singleton or empty set.

Assumption 1 is equivalent to deadbeat observability for linear systems. Below result formalizes this.

Theorem 1 Linear system (4) is deadbeat observable if and only if Assumption 1 holds.

Proof. Let $\mathcal{S}_{k}$ for $k=0,1, \ldots$ be defined as in Lemma 1]. Note then that $[x]_{0}=x+\mathcal{S}_{0}$. We claim that the following holds

$$
[x]_{k}=\left\{\begin{array}{ccc}
x+\mathcal{S}_{k} & \text { for } & x \in \mathcal{R}\left(A^{k}\right) \\
\emptyset & \text { for } & x \notin \mathcal{R}\left(A^{k}\right)
\end{array}\right.
$$

for all $k$. We employ induction to establish our claim. Suppose (7) holds for some $k$. Then we can write

$$
\begin{aligned}
{[x]_{k}^{+} } & =A\left[A^{-1} x\right]_{k} \\
& =A\left[A^{-1} x \cap \mathcal{R}\left(A^{k}\right)\right]_{k} .
\end{aligned}
$$

Note that $A^{-1} x \cap \mathcal{R}\left(A^{k}\right) \neq \emptyset$ if and only if $x \in \mathcal{R}\left(A^{k+1}\right)$. Since $[x]_{k+1}=[x]_{k}^{+} \cap$ $[x]_{0}$, we deduce that $[x]_{k+1}=\emptyset$ for $x \notin \mathcal{R}\left(A^{k+1}\right)$. Otherwise if $x \in \mathcal{R}\left(A^{k+1}\right)$ then there exists some $\eta \in \mathcal{R}\left(A^{k}\right)$ such that $A \eta=x$. Using this $\eta$ we can construct the equality $A^{-1} x=\eta+\mathcal{N}(A)$ and we can write

$$
\begin{aligned}
{[x]_{k+1} } & =[x]_{k}^{+} \cap[x]_{0} \\
& =A\left[A^{-1} x\right]_{k} \cap[x]_{0} \\
& =A[\eta+\mathcal{N}(A)]_{k} \cap[x]_{0} \\
& =A\left(\eta+\left(\mathcal{N}(A) \cap \mathcal{R}\left(A^{k}\right)\right)+\mathcal{S}_{k}\right) \cap[x]_{0} \\
& =\left(A \eta+A \mathcal{S}_{k}\right) \cap\left(x+S_{0}\right) \\
& =\left(x+A \mathcal{S}_{k}\right) \cap\left(x+\mathcal{S}_{0}\right) \\
& =x+\left(A \mathcal{S}_{k} \cap \mathcal{S}_{0}\right) \\
& =x+\mathcal{S}_{k+1} .
\end{aligned}
$$

Since (7) holds for $k=0$, our claim is valid.

Now suppose that the system is deadbeat observable. Then by (7) we see that Assumption 1 holds with $p=n+1$ thanks to Lemma 1. If however the system is not deadbeat observable, then by $\operatorname{Remark} 1 \operatorname{dim} \mathcal{S}_{k} \geq 1$ for all $k$. We deduce by (7) therefore that $[0]_{k}$ can never be singleton nor is it empty. Hence Assumption 1 must fail.

Assumption 2 Given $x, \hat{x} \in \mathcal{X}$ and $k ; \hat{x} \in[x]_{k}^{+}$implies $[\hat{x}]_{k}^{+}=[x]_{k}^{+}$.

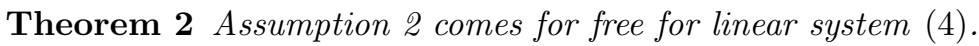


Proof. Evident.

Last we let $[x]_{-1}^{+}:=\mathcal{X}$ and define map $\pi: \mathcal{X} \times \mathcal{Y} \rightarrow\{-1,0,1, \ldots, p-2\}$ as $\pi(\hat{x}, y):=\max \{-1,0,1, \ldots, p-2\} \quad$ subject to $[\hat{x}]_{\pi(\hat{x}, y)}^{+} \cap h^{-1}(y) \neq \emptyset$

where $p$ is as in Assumption 1 .

\section{The result}

Below is our main theorem.

Theorem 3 Suppose Assumptions [1] hold. Then system

$$
\hat{x}^{+} \in f\left([\hat{x}]_{\pi(\hat{x}, y)}^{+} \cap h^{-1}(y)\right)
$$

is a deadbeat observer for system (2).

Proof. We claim the following

$$
\hat{x} \in[x]_{\ell-1}^{+} \Longrightarrow \hat{x}^{+} \in[f(x)]_{\ell}^{+}
$$

for all $\ell \in\{0,1, \ldots, p-1\}$. Let us prove our claim. Note that $\hat{x} \in[x]_{\ell-1}^{+}$yields $[\hat{x}]_{\ell-1}^{+}=[x]_{\ell-1}^{+}$by Assumption 2. Since $[x]_{\ell-1}^{+} \neq \emptyset$ we have $[x]_{\ell-1}^{+} \cap[x]_{0} \neq \emptyset$ and, consequently, $[\hat{x}]_{\ell-1}^{+} \cap[x]_{0} \neq \emptyset$. Remark 2 then yields $[\hat{x}]_{\pi(\hat{x}, h(x))}^{+} \subset[\hat{x}]_{\ell-1}^{+}$. Starting from (8) we can proceed as

$$
\begin{aligned}
\hat{x}^{+} & \in f\left([\hat{x}]_{\pi(\hat{x}, y)}^{+} \cap h^{-1}(y)\right) \\
& =f\left([\hat{x}]_{\pi(\hat{x}, h(x))}^{+} \cap h^{-1}(h(x))\right) \\
& \subset f\left([\hat{x}]_{\ell-1}^{+} \cap[x]_{0}\right) \\
& =f\left([x]_{\ell-1}^{+} \cap[x]_{0}\right) \\
& =f\left([x]_{\ell}\right) \\
& \subset f\left(\left[f^{-1}(f(x))\right]_{\ell}\right) \\
& =[f(x)]_{\ell}^{+} .
\end{aligned}
$$

Hence (9) holds. In particular, (10) gives us

$$
\hat{x} \in[x]_{\ell-1}^{+} \Longrightarrow \hat{x}^{+} \in f\left([x]_{\ell}\right)
$$

for all $\ell \in\{0,1, \ldots, p-1\}$. Note that $\hat{x} \in[x]_{-1}^{+}$holds for all $x, \hat{x}$. Therefore (9) and Remark 2 imply the existence of $\ell^{*} \in\{0,1, \ldots, p-1\}$ such that

$$
\psi(k, \hat{x}, x) \in[\phi(k, x)]_{p-2}^{+}
$$

for all $k \geq \ell^{*}$. Also, Assumption 1yields us

$$
[\phi(k, x)]_{p-1}=\phi(k, x)
$$


for all $k \geq p-1$. Combining (11), (12), and (13) we can write

$$
\psi(k, \hat{x}, x)=\phi(k, x)
$$

for all $k \geq p$. Hence the result.

Corollary 1 Consider linear system (4) with $A \in \mathbb{R}^{n \times n}$ and $C \in \mathbb{R}^{1 \times n}$. Suppose pair $(C, A)$ is observabl艇. Let $\mathcal{S}_{k}$ for $k=0,1, \ldots$ be defined as in Lemma 1, Then system

$$
\hat{x}^{+}=A\left(\left(\hat{x}+A \mathcal{S}_{n-2}\right) \cap\left(x+\mathcal{S}_{0}\right)\right)
$$

is a deadbeat observer for system (4).

\section{System with input}

In this section we look at the case where the evolution of system to be observed is dependent not only on the initial condition but also on some exogenous signal, which we call the input. To construct a deadbeat observer for such system we again make use of sets.

Consider the system

$$
\begin{aligned}
x^{+} & =f(x, u) \\
y & =h(x)
\end{aligned}
$$

where $u \in \mathcal{U} \subset \mathbb{R}^{q}$ is the input or some known disturbance (e.g. time). Let $\mathbf{u}=\left(u_{0}, u_{1}, \ldots\right), u_{k} \in \mathcal{U}$, denote an input sequence. The solution of system (14) at time $k$, starting at initial condition $x \in \mathcal{X}$, and having evolved under the influence of input sequence $\mathbf{u}$ is denoted by $\phi(k, x, \mathbf{u})$. Note that $\phi(0, x, \mathbf{u})=x$ and $\phi(k+1, x, \mathbf{u})=f\left(\phi(k, x, \mathbf{u}), u_{k}\right)$ for all $x, \mathbf{u}$, and $k$.

Now consider the following cascade system

$$
\begin{aligned}
& x^{+}=f(x, u) \\
& \hat{x}^{+} \in g(\hat{x}, h(x), u)
\end{aligned}
$$

We denote a solution of subsystem (15b) by $\psi(k, \hat{x}, x, \mathbf{u})$. We then have $\psi(0, \hat{x}, x, \mathbf{u})=\hat{x}$ and $\psi(k+1, \hat{x}, x, \mathbf{u}) \in g\left(\psi(k, \hat{x}, x, \mathbf{u}), h(\phi(k, x, \mathbf{u})), u_{k}\right)$ for all $x, \hat{x}, \mathbf{u}$, and $k$.

Definition 3 Given $g: \mathcal{X} \times \mathcal{Y} \times \mathcal{U} \rightrightarrows \mathcal{X}$, system

$$
\hat{x}^{+} \in g(\hat{x}, y, u)
$$

is said to be a deadbeat observer for system (14) if there exists $p \geq 1$ such that solutions of system (15) satisfy

$$
\psi(k, \hat{x}, x, \mathbf{u})=\phi(k, x, \mathbf{u})
$$

for all $x, \hat{x}, \mathbf{u}$, and $k \geq p$.

\footnotetext{
${ }^{1}$ That is, rank $\left[C^{T} A^{T} C^{T} \ldots A^{(n-1) T} C^{T}\right]=n$.
} 
How to define sets $[x]_{k}$ and $[x]_{k}^{+}$for system (14) is obvious. We again let

$$
[x]_{0}:=h^{-1}(h(x)) \text {. }
$$

and (for $k=0,1, \ldots$ )

$$
[x]_{k+1}:=[x]_{k}^{+} \cap[x]_{0}
$$

this time with

$$
[x]_{k}^{+}:=\bigcup_{f(\eta, u)=x} f\left([\eta]_{k}, u\right) .
$$

The following result is a generalization of Theorem 3 . (The demonstration is parallel to that of Theorem 3 and hence omitted.)

Theorem 4 Suppose Assumptions 1 2 hold. Then system

$$
\hat{x}^{+} \in f\left([\hat{x}]_{\pi(\hat{x}, y)}^{+} \cap h^{-1}(y), u\right)
$$

is a deadbeat observer for system (14).

\section{$7 \quad$ Examples}

Here, for two third order nonlinear systems, we construct deadbeat observers. In the first example we study a simple autonomous homogeneous system and show that the construction yields a homogeneous observer. Hence our method may be thought to be somewhat natural in the vague sense that the observer it generates inherits certain intrinsic properties of the system. In the second example we aim to provide a demonstration on observer construction for a system with input.

\section{$7.1 \quad$ Homogeneous system}

Consider system (2) with

$$
f(x):=\left[\begin{array}{c}
x_{2} \\
x_{3}^{1 / 3} \\
x_{1}^{3}+x_{2}^{3}
\end{array}\right] \quad \text { and } \quad h(x):=x_{1}
$$

where $x=\left[\begin{array}{lll}x_{1} & x_{2} & x_{3}\end{array}\right]^{T}$. Let $\mathcal{X}=\mathbb{R}^{3}$ and $\mathcal{Y}=\mathbb{R}$. If we let dilation $\Delta_{\lambda}$ be

$$
\Delta_{\lambda}:=\left[\begin{array}{ccc}
\lambda & 0 & 0 \\
0 & \lambda & 0 \\
0 & 0 & \lambda^{3}
\end{array}\right]
$$

with $\lambda \in \mathbb{R}$, then we realize that

$$
f\left(\Delta_{\lambda} x\right)=\Delta_{\lambda} f(x) \text { and } h\left(\Delta_{\lambda} x\right)=\lambda h(x) .
$$


That is, the system is homogeneous [10] with respect to dilation $\Delta$. Before describing the relevant sets $[x]_{k}$ and $[x]_{k}^{+}$we want to mention that $f$ is bijective and its inverse is

$$
f^{-1}(x)=\left[\begin{array}{c}
\left(x_{3}-x_{1}^{3}\right)^{1 / 3} \\
x_{1} \\
x_{2}^{3}
\end{array}\right]
$$

Since $h(x)=x_{1}$ we can write

$$
[x]_{0}=\left\{\left[\begin{array}{c}
x_{1} \\
\alpha \\
\beta
\end{array}\right]: \alpha, \beta \in \mathbb{R}\right\}
$$

By (16) we can then proceed as

$$
\begin{aligned}
{[x]_{0}^{+} } & =f\left(\left[f^{-1}(x)\right]_{0}\right) \\
& =f\left(\left\{\left[\begin{array}{c}
\left(x_{3}-x_{1}^{3}\right)^{1 / 3} \\
\gamma \\
\delta
\end{array}\right]: \gamma, \delta \in \mathbb{R}\right\}\right) \\
& =\left\{f\left(\left[\begin{array}{c}
\left(x_{3}-x_{1}^{3}\right)^{1 / 3} \\
\gamma \\
\delta
\end{array}\right]\right): \gamma, \delta \in \mathbb{R}\right\} \\
& =\left\{\left[\begin{array}{c}
\gamma \\
\delta^{1 / 3} \\
x_{3}-x_{1}^{3}+\gamma^{3}
\end{array}\right]: \gamma, \delta \in \mathbb{R}\right\}
\end{aligned}
$$

Recall that $[x]_{1}=[x]_{0}^{+} \cap[x]_{0}$. Therefore intersecting sets (17) and (18) we obtain

$$
[x]_{1}=\left\{\left[\begin{array}{c}
x_{1} \\
\alpha \\
x_{3}
\end{array}\right]: \alpha \in \mathbb{R}\right\}
$$

We can now construct $[x]_{1}^{+}$as

$$
\begin{aligned}
{[x]_{1}^{+} } & =f\left(\left[f^{-1}(x)\right]_{1}\right) \\
& =f\left(\left\{\left[\begin{array}{c}
\left(x_{3}-x_{1}^{3}\right)^{1 / 3} \\
\gamma \\
x_{2}^{3}
\end{array}\right]: \gamma \in \mathbb{R}\right\}\right) \\
& =\left\{f\left(\left[\begin{array}{c}
\left(x_{3}-x_{1}^{3}\right)^{1 / 3} \\
\gamma \\
x_{2}^{3}
\end{array}\right]\right): \gamma \in \mathbb{R}\right\} \\
& =\left\{\left[\begin{array}{c}
\gamma \\
x_{2} \\
x_{3}-x_{1}^{3}+\gamma^{3}
\end{array}\right]: \gamma \in \mathbb{R}\right\}
\end{aligned}
$$


Now note that sets (17) and (19) intersect at a single point. In particular, $[x]_{2}=[x]_{1}^{+} \cap[x]_{0}=x$. Therefore Assumption 1 is satisfied with $p=3$. Observe also that

$$
\begin{aligned}
{[\hat{x}]_{1}^{+} \cap h^{-1}(y) } & =\left\{\left[\begin{array}{c}
\gamma \\
\hat{x}_{2} \\
\hat{x}_{3}-\hat{x}_{1}^{3}+\gamma^{3}
\end{array}\right]: \gamma \in \mathbb{R}\right\} \cap\left\{\left[\begin{array}{c}
y \\
\alpha \\
\beta
\end{array}\right]: \alpha, \beta \in \mathbb{R}\right\} \\
& =\left[\begin{array}{c}
y \\
\hat{x}_{2} \\
\hat{x}_{3}-\hat{x}_{1}^{3}+y^{3}
\end{array}\right]
\end{aligned}
$$

which means that $\pi(\hat{x}, y)=p-2=1$ for all $\hat{x}$ and $y$. The dynamics of the deadbeat observer then read

$$
\begin{aligned}
\hat{x}^{+} & =f\left([\hat{x}]_{1}^{+} \cap h^{-1}(y)\right) \\
& =\left[\begin{array}{c}
\hat{x}_{2} \\
\left(\hat{x}_{3}-\hat{x}_{1}^{3}+y^{3}\right)^{1 / 3} \\
\hat{x}_{2}^{3}+y^{3}
\end{array}\right]
\end{aligned}
$$

We finally notice that

$$
f\left(\left[\Delta_{\lambda} \hat{x}\right]_{1}^{+} \cap h^{-1}(\lambda y)\right)=\Delta_{\lambda} f\left([\hat{x}]_{1}^{+} \cap h^{-1}(y)\right) .
$$

That is, the deadbeat observer also is homogeneous with respect to dilation $\Delta$.

\subsection{System with input}

Our second example is again a third order system, this time however with an input. Consider system (14) with

$$
f(x, u):=\left[\begin{array}{c}
x_{1} x_{2} x_{3} \\
x_{3} / x_{1} \\
\sqrt{x_{1} x_{2} u}
\end{array}\right] \quad \text { and } \quad h(x):=x_{1} .
$$

Let $\mathcal{X}=\mathbb{R}_{>0}^{3}, \mathcal{Y}=\mathbb{R}_{>0}$, and $\mathcal{U}=\mathbb{R}_{>0}$. Let us construct the relevant sets $[x]_{k}$ and $[x]_{k}^{+}$. We begin with $[x]_{0}$.

$$
[x]_{0}=\left\{\left[\begin{array}{c}
x_{1} \\
\alpha \\
\beta
\end{array}\right]: \alpha, \beta>0\right\}
$$

Note that $f$ satisfies the following

$$
f\left(\left[\begin{array}{c}
x_{1} u /\left(x_{2} x_{3}^{2}\right) \\
x_{2} x_{3}^{4} /\left(x_{1} u^{2}\right) \\
x_{1} u / x_{3}^{2}
\end{array}\right], u\right)=x
$$


for all $x$ and $u$. Hence we can write

$$
\begin{aligned}
{[x]_{0}^{+} } & =\bigcup_{u \in \mathcal{U}} f\left(\left[\begin{array}{c}
x_{1} u /\left(x_{2} x_{3}^{2}\right) \\
x_{2} x_{3}^{4} /\left(x_{1} u^{2}\right) \\
x_{1} u / x_{3}^{2}
\end{array}\right]_{0}, u\right) \\
& =\bigcup_{u \in \mathcal{U}} f\left(\left\{\left[\begin{array}{c}
x_{1} u /\left(x_{2} x_{3}^{2}\right) \\
\gamma \\
\delta
\end{array}\right]: \gamma, \delta>0\right\}, u\right) \\
& =\bigcup_{u \in \mathcal{U}} f\left(\left\{\left[\begin{array}{c}
x_{1} u /\left(x_{2} x_{3}^{2}\right) \\
x_{2} x_{3}^{4} \gamma /\left(x_{1} u^{2}\right) \\
x_{1} u \delta / x_{3}^{2}
\end{array}\right]: \gamma, \delta>0\right\}, u\right) \\
& =\bigcup_{u \in \mathcal{U}}\left\{f\left(\left[\begin{array}{c}
x_{1} u /\left(x_{2} x_{3}^{2}\right) \\
x_{2} x_{3}^{4} \gamma /\left(x_{1} u^{2}\right) \\
x_{1} u \delta / x_{3}^{2}
\end{array}\right], u\right): \gamma, \delta>0\right\} \\
& =\left\{\left[\begin{array}{c}
x_{1} \gamma \delta \\
x_{2} \delta \\
x_{3} \sqrt{\gamma}
\end{array}\right] \begin{array}{c}
: \gamma, \delta>0 \\
:
\end{array}\right.
\end{aligned}
$$

Since $[x]_{1}=[x]_{0}^{+} \cap[x]_{0}$, intersecting sets (20) and (21) we obtain

$$
[x]_{1}=\left\{\left[\begin{array}{c}
x_{1} \\
x_{2} / \alpha^{2} \\
x_{3} \alpha
\end{array}\right]: \alpha>0\right\}
$$

We can now construct $[x]_{1}^{+}$as

$$
\begin{aligned}
{[x]_{1}^{+} } & =\bigcup_{u \in \mathcal{U}} f\left(\left[\begin{array}{c}
x_{1} u /\left(x_{2} x_{3}^{2}\right) \\
x_{2} x_{3}^{4} /\left(x_{1} u^{2}\right) \\
x_{1} u / x_{3}^{2}
\end{array}\right]_{1}, u\right) \\
& =\bigcup_{u \in \mathcal{U}} f\left(\left\{\left[\begin{array}{c}
x_{1} u /\left(x_{2} x_{3}^{2}\right) \\
x_{2} x_{3}^{4} /\left(x_{1} u^{2} \gamma^{2}\right) \\
x_{1} u \gamma / x_{3}^{2}
\end{array}\right]: \gamma>0\right\}, u\right) \\
& =\left\{\left[\begin{array}{c}
x_{1} / \gamma \\
x_{2} \gamma \\
x_{3} / \gamma
\end{array}\right]: \gamma>0\right\}
\end{aligned}
$$

Now note that sets (20) and (22) intersect at a single point. In particular, $[x]_{2}=[x]_{1}^{+} \cap[x]_{0}=x$. Therefore Assumption 11 is satisfied with $p=3$. Observe also that

$$
\begin{aligned}
{[\hat{x}]_{1}^{+} \cap h^{-1}(y) } & =\left\{\left[\begin{array}{c}
\hat{x}_{1} / \gamma \\
\hat{x}_{2} \gamma \\
\hat{x}_{3} / \gamma
\end{array}\right]: \gamma>0\right\} \cap\left\{\left[\begin{array}{l}
y \\
\alpha \\
\beta
\end{array}\right]: \alpha, \beta>0\right\} \\
& =\left[\begin{array}{c}
y \\
\hat{x}_{1} \hat{x}_{2} / y \\
\hat{x}_{3} y / \hat{x}_{1}
\end{array}\right]
\end{aligned}
$$


which means that $\pi(\hat{x}, y)=p-2=1$ for all $\hat{x}$ and $y$. The dynamics of the deadbeat observer then read

$$
\begin{aligned}
\hat{x}^{+} & =f\left([\hat{x}]_{1}^{+} \cap h^{-1}(y), u\right) \\
& =\left[\begin{array}{c}
\hat{x}_{2} \hat{x}_{3} y \\
\hat{x}_{3} / \hat{x}_{1} \\
\sqrt{\hat{x}_{1} \hat{x}_{2} u}
\end{array}\right]
\end{aligned}
$$

\section{An algorithm for deadbeat gain}

In this section we provide an algorithm to compute the deadbeat observer gain for a linear system with scalar output. (The algorithm directly follows from Corollary [1) Namely, given an observable pair $(C, A)$ with $C \in \mathbb{R}^{1 \times n}$ and $A \in \mathbb{R}^{n \times n}$, we provide a procedure to compute the gain $L \in \mathbb{R}^{n \times 1}$ that renders matrix $A-L C$ nilpotent. Below we let null(.) be some function such that, given matrix $M \in \mathbb{R}^{m \times n}$ whose dimension of null space is $k$, null $(M)$ is some $n \times k$ matrix whose columns span the null space of $M$.

Algorithm 1 Given $C \in \mathbb{R}^{1 \times n}$ and $A \in \mathbb{R}^{n \times n}$, the following algorithm generates deadbeat gain $L \in \mathbb{R}^{n \times 1}$.

$$
\begin{aligned}
& X=\operatorname{null}(C) \\
& \text { for } \quad i=1: n-2 \\
& \quad X=\operatorname{null}\left(\left[\begin{array}{c}
C \\
\operatorname{null}\left((A X)^{T}\right)^{T}
\end{array}\right]\right) \\
& \text { end } \\
& L_{\text {pre }}=A X \\
& L=\frac{A L_{\text {pre }}}{C L_{\text {pre }}}
\end{aligned}
$$

For the interested reader we below give a MATLAB code. Exploiting Algorithm 1, this code generates a function (which we named dbLfun) whose inputs are matrices $C$ and $A$. The output of the function, as its name indicates, is the deadbeat gain $L$.



One can also use the built-in MATLAB function acker to compute the deadbeat gain. We can therefore compare dbLfun with acker via a numerical experiment. Table 1 gives the experimental results. Number $n$ is the dimension of the system 
Table 1: Percentages of cases where dbLfun performed better than acker.

\begin{tabular}{|c|c|c|c|c|c|c|c|}
\hline $\mathrm{n}=3$ & $\mathrm{n}=4$ & $\mathrm{n}=5$ & $\mathrm{n}=6$ & $\mathrm{n}=7$ & $\mathrm{n}=8$ & $\mathrm{n}=9$ & $\mathrm{n}=10$ \\
\hline$\% 51$ & $\% 60$ & $\% 67$ & $\% 74$ & $\% 80$ & $\% 85$ & $\% 87$ & $\% 91$ \\
\hline
\end{tabular}

(that is, the number of columns of $A$ matrix) and the numbers at the bottom row are the percentages of the cases (among $10^{4}$ random trials for each $n$ ) in which dbLfun performed better than acker. How we determine which one is better in a given case is as follows. Given pair $(C, A)$, we let $L_{1}$ be the gain resulting from dbLfun (C,A) and $L_{2}$ be the gain given by acker (A', C', zeros (n,1))'. Then we compare norms $\left|\left(A-L_{1} C\right)^{n}\right|$ and $\left|\left(A-L_{2} C\right)^{n}\right|$, neither of which is zero due to round-off errors. The function yielding the smaller norm is considered to be better.

\section{Conclusion}

For nonlinear systems a method to construct a deadbeat observer is proposed. The resultant observer can be considered as a generalization of the linear deadbeat observer. The construction makes use of sets that are generated iteratively. Through such iterations, observers are derived for two academic examples. Also, for computing the deadbeat gain for a linear system with scalar output, an algorithm that works no worse than an already existing one is given.

\section{References}

[1] G. Besançon. Remarks on nonlinear adaptive observer design. Systems $\&$ Control Letters, 41(4):271-280, 2000.

[2] A. Emami-Naeini and G.F. Franklin. Deadbeat control and tracking of discrete-time systems. IEEE Transactions on Automatic Control, 27(1):176-181, 1982.

[3] P.A. Fuhrmann and J. Trumpf. On observability subspaces. International Journal of Control, 79(10):1157-1195, 2006.

[4] S.T. Glad. Observability and nonlinear dead beat observers. In Proc. of the 22nd IEEE Conference on Decision and Control, pages 800-802, 1983.

[5] I. Karafyllis and C. Kravaris. On the observer problem for discrete-time control systems. IEEE Transactions on Automatic Control, 52(1):12-25, 2007.

[6] P.D. Lax. Linear Algebra. Wiley-Interscience, 1996. 
[7] F.L. Lewis. A general Riccati equation solution to the deadbeat control problem. IEEE Transactions on Automatic Control, 27(1):186-188, 1982.

[8] D.G. Luenberger. Observing the state of a linear system. IEEE Transactions on Military Electronics, 8(2):74-80, 1964.

[9] P.E. Moraal and J.W. Grizzle. Observer design for nonlinear systems with discrete-time measurements. IEEE Transactions on Automatic Control, 40(3):395-404, 1995.

[10] M. Rinehart, M. Dahleh, and I. Kolmanovsky. Value iteration for (switched) homogeneous systems. IEEE Transactions on Automatic Control, 54(6):1290-1294, 2009.

[11] J.S. Shamma and K.-Y. Tu. Set-valued observers and optimal disturbance rejection. IEEE Transactions on Automatic Control, 44(2):253-264, 1999.

[12] K. Sugimoto, A. Inoue, and S. Masuda. A direct computation of state deadbeat feedback gains. IEEE Transactions on Automatic Control, 38(8):1283$1284,1993$.

[13] M.E. Valcher and J.C. Willems. Dead beat observer synthesis. Systems $\mathcal{E}$ Control Letters, 37(5):285-292, 1999.

[14] K.C. Wong and W.M. Wonham. On the computation of observers in discrete-event systems. Discrete Event Dynamic Systems - Theory and Applications, 14(1):55-107, 2004. 